\title{
Bounds on third Hankel determinant for close-to-convex functions
}

\author{
J. K. Prajapat
}

Department of Mathematics, Central University of Rajasthan, India email: jkprajapat@curaj.ac.in

Alok Singh

Department of Mathematics, Central University of Rajasthan, India email: aloksingh@curaj.ac.in

\section{Deepak Bansal}

Department of Mathematics, Govt. College of Engineering and

Technology, India email: deepakbansal_79@yahoo.com

\author{
Ambuj K. Mishra \\ Department of Mathematics, \\ GLA University, India \\ email: Ambuj math@rediffmail.com
}

\begin{abstract}
In this paper, we have obtained upper bound on third Hankel determinant for the functions belonging to the class of close-to-convex functions.
\end{abstract}

\section{Introduction}

Let $\mathcal{H}(\mathbb{U})$ denote the class of functions which are analytic in the open unit disk $\mathbb{U}=\{z:|z|<1\}$. Let $\mathcal{A}$ be the class of all functions $\mathrm{f} \in \mathcal{H}(\mathbb{U})$ which are normalized by $f(0)=0, f^{\prime}(0)=1$ and have the following form:

$$
f(z)=z+a_{2} z^{2}+a_{3} z^{3}+\ldots, \quad z \in \mathbb{U} .
$$

2010 Mathematics Subject Classification: 30C45

Key words and phrases: analytic functions, univalent function, close-to-convex function, Fekete-Szego functional, Hankel determinant 
We denote by $\mathcal{S}$ the subclass of $\mathcal{A}$ consisting of all functions in $\mathcal{A}$ which are also univalent in $\mathbb{U}$. Let $\mathcal{P}$ be the class of all functions $p \in \mathcal{H}(\mathbb{U})$ satisfying $p(0)=1$ and $\mathfrak{R}(p(z))>0$. The function $p \in \mathcal{P}$ have the following form:

$$
p(z)=1+c_{1} z+c_{2} z^{2}+\ldots, \quad z \in \mathbb{U} .
$$

Further, a function $f \in \mathcal{A}$ is said to belong to the class $\mathcal{S}^{*}$ of starlike functions in $\mathbb{U}$, if it satisfies the following inequality:

$$
\mathfrak{R}\left(\frac{z f^{\prime}(z)}{f(z)}\right)>0, \quad z \in \mathbb{U} .
$$

Moreover, a function $f \in \mathcal{A}$ is said to belong to the class $\mathcal{C}$ of close-to-convex functions in $\mathbb{U}$, if there exist a function $g \in \mathcal{S}^{*}$, such that the following inequality holds:

$$
\Re\left(\frac{z f^{\prime}(z)}{g(z)}\right)>0, \quad z \in \mathbb{U} .
$$

The class of close-to-convex functions was introduced by Kaplan [9]. In [16], Noonan and Thomas studied the $\mathrm{q}^{\text {th }}$ Hankel determinants $\mathrm{H}_{\mathrm{q}}(\mathrm{n})$ of functions $\mathrm{f} \in \mathcal{A}$ of the form (1) for $\mathrm{q} \geq 1$, which is defined by

$$
H_{q}(n)=\left|\begin{array}{cccc}
a_{n} & a_{n+1} & \ldots & a_{n+q-1} \\
a_{n+1} & \ldots & \ldots & \vdots \\
\vdots & \vdots & \vdots & \vdots \\
a_{n+q-1} & \ldots & \ldots & a_{n+2(q-1)}
\end{array}\right| \quad\left(a_{1}=1\right) .
$$

The Hankel determinants $H_{q}(n)$ have been investigated by several authors to study its rate of growth as $n \rightarrow \infty$ and to determine bounds on it for specific values of $q$ and $n$. For example, Pommerenke [22] proved that the Hankel determinants of univalent functions satisfy $\left|\mathrm{H}_{\mathrm{q}}(\mathrm{n})\right|<\mathrm{Kn}^{-\left(\frac{1}{2}+\beta\right) \mathrm{q}+\frac{3}{2}}(\mathrm{n}=$ $1,2, \ldots, q=2,3, \ldots)$, where $\beta>1 / 4000$ and $K$ depends only on $q$. Later, Hayman [8] proved that $\left|H_{2}(n)\right|<A n^{1 / 2}(n=1,2, \ldots ; A$ is an absolute constant $)$ for areally mean univalent functions. Pommerenke [21] investigated the Hankel determinant of areally mean $p$-valent functions, univalent functions as well as of starlike functions. Ehrenborg studied Hankel determinant of the exponential polynomials [6] and Noor studied Hankel determinant for Bazilevic functions in [18] and for functions with bounded boundary rotations in $[17,19]$ also for close-to-convex functions in [20].

A classical theorem of Fekete and Szegö [7] considered the second Hankel determinant $\mathrm{H}_{2}(1)=\mathrm{a}_{3}-\mathrm{a}_{2}^{2}$ for univalent functions. They made an early 
study for the estimate of well known Fekete-Szegö functional $\left|\mathrm{a}_{3}-\mu \mathrm{a}_{2}^{2}\right|$ when $\mu$ is real. Jenteng [12] investigated the sharp upper bound for second Hankel determinant $\left|\mathrm{H}_{2}(2)\right|=\left|\mathrm{a}_{2} \mathrm{a}_{4}-\mathrm{a}_{3}^{2}\right|$ for univalent functions whose derivative has positive real part. Recently, Lee et al. [13] have obtained bounds on $\left|\mathrm{H}_{2}(2)\right|$ for functions belonging to the subclasses of Ma-Minda starlike and convex functions. Further Bansal [2] have obtained bounds on $\left|\mathrm{H}_{2}(2)\right|$ for some new class of analytic functions. Recently, Babalola [1], Raza and Malik [24] and Bansal et al. [3] have studied third Hankel determinant $\mathrm{H}_{3}(1)$, for various classes of analytic and univalent functions. In the present paper we investigate the upper bound on $\left|\mathrm{H}_{3}(1)\right|$ for the functions belonging to the class of closeto-convex functions $\mathcal{K}$ defined by (4). To derive our results, we shall need the following Lemmas:

Lemma 1 (Carathéodory's Lemma [4], see also [5, p. 41]). Let the function $p \in \mathcal{P}$ be given by the series then the sharp estimate $\left|\mathrm{c}_{n}\right| \leq 2, n=1,2, \cdots$ holds. The inequality is sharp for each $\mathrm{n}$.

Lemma 2 (cf. [14, p. 254], see also [15]). Let the function $\mathrm{p} \in \mathcal{P}$ be given by (2), then

$$
2 c_{2}=c_{1}^{2}+x\left(4-c_{1}^{2}\right)
$$

for some $\mathrm{x},|\mathrm{x}| \leq 1$, and

$$
4 c_{3}=c_{1}^{3}+2 c_{1}\left(4-c_{1}^{2}\right) x-c_{1}\left(4-c_{1}^{2}\right) x^{2}+2\left(4-c_{1}^{2}\right)\left(1-|x|^{2}\right) z
$$

for some $z,|z| \leq 1$.

Lemma 3 ([5, p. 44]). If $\mathrm{f} \in \mathcal{S}^{*}$ be given by (1), then $\left|\mathrm{a}_{\mathrm{n}}\right| \leq \mathrm{n}(\mathrm{n}=$ $2,3, \ldots)$. Strict inequality holds for all $\mathrm{n}$ unless $\mathrm{f}$ is rotation of the Koebe function $\mathrm{k}(z)=z /(1-z)^{2}$.

Lemma 4 ([23]). If $\mathrm{f} \in \mathcal{C}$ be given by (1), then $\left|\mathrm{a}_{\mathrm{n}}\right| \leq \mathrm{n}(\mathrm{n}=2,3, \ldots)$. Equality holds for all $\mathrm{n}$ when $\mathrm{f}$ is rotation of the Koebe function.

Lemma 5 ([10]). If $\mathrm{f} \in \mathcal{S}^{*}$ be given by (1), then for any real number $\mu$, we have

$$
\left|a_{3}-\mu a_{2}^{2}\right| \leq \begin{cases}3-4 \mu, & \text { if } \mu \leq \frac{1}{2} \\ 1, & \text { if } \frac{1}{2} \leq \mu \leq 1 \\ 4 \mu-3, & \text { if } \mu \geq 1\end{cases}
$$

Lemma 6 ([11]). If $\mathrm{f} \in \mathcal{C}$ be given by (1), then $\left|\mathrm{a}_{3}-\mathrm{a}_{2}^{2}\right| \leq 1$. There is a function in $\mathcal{C}$ such that equality holds. 
Lemma 7 ([12]). If $\mathrm{f} \in \mathcal{S}^{*}$ be given by (1), then $\left|\mathrm{a}_{2} \mathrm{a}_{4}-\mathrm{a}_{3}^{2}\right| \leq 1$. Equality is attended for the the Koebe function.

Lemma 8 ([1]). If $\mathrm{f} \in \mathcal{S}^{*}$ be given by (1), then $\left|\mathrm{a}_{2} \mathrm{a}_{3}-\mathrm{a}_{4}\right| \leq 2$. Equality is attained by Koebe function.

\section{Main results}

Our first main result is contained in the following theorem:

Theorem 1 Let the function $\mathrm{f} \in \mathcal{C}$ be given by (1), then

$$
\left|a_{2} a_{3}-a_{4}\right| \leq 3 \text {. }
$$

Proof. Let the function $f \in \mathcal{C}$ be given by (6), then from the definition, we have

$$
z f^{\prime}(z)=g(z) p(z), \quad z \in \mathbb{U}
$$

for $p(z) \in \mathcal{P}$. The function $g(z)$ in $(7)$ is a starlike function and let it have the form $g(z)=z+b_{2} z^{2}+b_{3} z^{3}+\ldots$. Substituting the valves of $f(z), g(z)$ and $p(z)$ and equating the coefficients, we get

$$
\begin{aligned}
& 2 a_{2}=b_{2}+c_{1} \\
& 3 a_{3}=b_{3}+b_{2} c_{1}+c_{2} \\
& 4 a_{4}=b_{4}+b_{3} c_{1}+b_{2} c_{2}+c_{3} .
\end{aligned}
$$

Now

$$
\begin{aligned}
& \left|a_{2} a_{3}-a_{4}\right|=\left|\frac{b_{2}+c_{1}}{2} \frac{b_{3}+b_{2} c_{1}+c_{2}}{3}-\frac{b_{4}+b_{3} c_{1}+b_{2} c_{2}+c_{3}}{4}\right| \\
& =\mid \frac{1}{4}\left(b_{2} b_{3}-b_{4}\right)-\frac{c_{1}}{12}\left(b_{3}-2 b_{2}^{2}\right)-\frac{1}{12} b_{2} b_{3}+\frac{1}{6} b_{2} c_{1}^{2} \\
& +\left(\frac{c_{1}}{6}-\frac{b_{2}}{12}\right) c_{2}-\frac{c_{3}}{4}
\end{aligned}
$$

Substituting values of $c_{2}$ and $c_{3}$ by Lemma 2 in (11), we get

$$
\begin{aligned}
\left|a_{2} a_{3}-a_{4}\right|= & \mid \frac{1}{4}\left(b_{2} b_{3}-b_{4}\right)-\frac{c_{1}}{12}\left(b_{3}-2 b_{2}^{2}\right)-\frac{1}{12} b_{2} b_{3} \\
& +\frac{1}{6} b_{2} c_{1}^{2}+\left(\frac{c_{1}}{6}-\frac{b_{2}}{12}\right) \frac{c_{1}^{2}+\left(4-c_{1}^{2}\right) x}{2} \\
& -\frac{c_{1}^{3}+2 c_{1}\left(4-c_{1}^{2}\right) x-c_{1}\left(4-c_{1}^{2}\right) x^{2}+2\left(4-c_{1}^{2}\right)\left(1-|x|^{2}\right) z}{16} \mid
\end{aligned}
$$




$$
\begin{aligned}
= & \mid \frac{1}{4}\left(b_{2} b_{3}-b_{4}\right)-\frac{c_{1}}{12}\left(b_{3}-2 b_{2}^{2}\right)-\frac{1}{12} b_{2} b_{3}+\frac{1}{48} c_{1}^{3}-\frac{1}{24} c_{1}\left(4-c_{1}^{2}\right) x+\frac{1}{8} b_{2} c_{1}^{2} \\
& -\frac{1}{24} b_{2}\left(4-c_{1}^{2}\right) x+\frac{1}{16} c_{1}\left(4-c_{1}^{2}\right) x^{2}-\frac{1}{8}\left(4-c_{1}^{2}\right)\left(1-|x|^{2}\right) z \mid
\end{aligned}
$$

By Lemma 1, we have $\left|c_{1}\right| \leq 2$. For convenience of notation, we take $c_{1}=c$ and we may assume without loss of generality that $c \in[0,2]$. Applying the triangle inequality with $\mu=|x|$ and using Lemma 3, Lemma 5 and Lemma 8, we obtain

$$
\begin{aligned}
\left|a_{2} a_{3}-a_{4}\right| \leq & \frac{1}{4}\left|b_{2} b_{3}-b_{4}\right|+\frac{1}{12} c\left|b_{3}-2 b_{2}^{2}\right|+\frac{1}{12}\left|b_{2}\right|\left|b_{3}\right|+\frac{1}{48} c^{3}+\frac{1}{8}\left|b_{2}\right| c^{2} \\
& +\frac{1}{24}\left(4-c^{2}\right)\left(c+\left|b_{2}\right|\right) \mu+\frac{c}{16}\left(4-c^{2}\right) \mu^{2}+\frac{1}{8}\left(4-c^{2}\right)\left(1-\mu^{2}\right) \\
\leq & \frac{3}{2}+\frac{5}{12} c+\frac{1}{8} c^{2}+\frac{1}{48} c^{3}+\frac{1}{24}\left(4-c^{2}\right)(c+2) \mu \\
& +\frac{1}{16}\left(4-c^{2}\right)(c-2) \mu^{2}=F_{1}(c, \mu) .
\end{aligned}
$$

Differentiating $F_{1}(c, \mu)$ partially with respect to $c$, we have

$$
\begin{aligned}
\frac{\partial F_{1}}{\partial c} & =\frac{5}{12}+\frac{c}{4}+\frac{c^{2}}{16}+\frac{\mu}{24}\left(4-3 c^{2}-4 c\right)+\frac{\mu^{2}}{16}\left(4-3 c^{2}+4 c\right) \\
& =\frac{1}{12}\left(5-\mu c^{2}\right)+\frac{c}{12}(3-2 \mu)+\frac{c^{2}}{16}+\frac{\mu}{24}\left(4-c^{2}\right)+\frac{\mu^{2}}{16}(2-c)(3 c+2)>0,
\end{aligned}
$$

for $c \in[0,2]$ and for any fixed $\mu$ with $\mu \in[0,1]$. Therefore $F_{1}(c, \mu)$ is an increasing function of $c$ on the closed interval $[0,2]$, and hence $F_{1}(c, \mu)$ attained its maximum value at $c=2$. Thus

$$
\max _{0 \leq c \leq 2} F_{1}(c, \mu)=F_{1}(2, \mu)=G_{1}(\mu) \text { (say). }
$$

From (12) and (13), we get $G_{1}(\mu)=3$, which is independent of $\mu$. Hence, the sharp upper bound of the functional $\left|a_{2} a_{3}-a_{4}\right|$ can be obtained by setting $c=2$ in (12), therefore

$$
\left|a_{2} a_{3}-a_{4}\right| \leq 3 .
$$

This completes the proof of Theorem 1 .

Theorem 2 Let the function $\mathrm{f} \in \mathcal{C}$ be given by (1), then

$$
\mathrm{H}_{2}(2)=\left|\mathrm{a}_{2} \mathrm{a}_{4}-\mathrm{a}_{3}^{2}\right| \leq \frac{85}{36} .
$$


Proof. Let $f \in \mathcal{C}$ of the form (1), then following the proof of Theorem 1, we get values of $a_{2}, a_{3}$ and $a_{4}$ given in (8)-(10). Using these values, we have

$$
\begin{aligned}
\left|a_{2} a_{4}-a_{3}^{2}\right|= & \left|\frac{b_{2}+c_{1}}{2} \cdot \frac{b_{4}+b_{3} c_{1}+b_{2} c_{2}+c_{3}}{4}-\left(\frac{b_{3}+b_{2} c_{1}+c_{2}}{3}\right)^{2}\right| \\
= & \mid \frac{1}{8} b_{2} b_{4}-\frac{7}{72} b_{2} b_{3} c_{1}+\frac{1}{8} b_{2}^{2} c_{2}+\frac{1}{8} b_{2} c_{3}+\frac{1}{8} b_{3} c_{1}^{2}-\frac{7}{72} b_{2} c_{1} c_{2} \\
& +\frac{1}{8} b_{4} c_{1}+\frac{1}{8} c_{1} c_{3}-\frac{1}{9} b_{3}^{2}-\frac{1}{9} b_{2}^{2} c_{1}^{2}-\frac{1}{9} c_{2}^{2}-\frac{2}{9} b_{3} c_{2} \mid \\
= & \mid \frac{1}{8}\left(b_{4}-b_{2} b_{3}\right) c_{1}+\frac{1}{8}\left(b_{3}-\frac{8}{9} b_{2}^{2}\right) c_{1}^{2}+\frac{1}{8}\left(b_{2} b_{4}-b_{3}^{2}\right) \\
& -\frac{2}{9}\left(b_{3}-\frac{9}{16} b_{2}^{2}\right) c_{2}+\frac{1}{36} b_{2} b_{3} c_{1} \\
& +\frac{1}{8} b_{2} c_{3}-\frac{7}{72} b_{2} c_{1} c_{2}+\frac{1}{8} c_{1} c_{3}+\frac{1}{72} b_{3}^{2}-\frac{1}{9} c_{2}^{2} \mid
\end{aligned}
$$

Substituting the values of $c_{2}$ and $c_{3}$ from Lemma 2 in above equation, we have

$$
\begin{aligned}
\left|a_{2} a_{4}-a_{3}^{2}\right|=\mid & \mid \frac{1}{8}\left(b_{4}-b_{2} b_{3}\right) c_{1}+\frac{1}{8}\left(b_{3}-\frac{8}{9} b_{2}^{2}\right) c_{1}^{2}+\frac{1}{8}\left(b_{2} b_{4}-b_{3}^{2}\right) \\
& -\frac{1}{9}\left(b_{3}-\frac{9}{16} b_{2}^{2}\right)\left(c_{1}^{2}+x\left(4-c_{1}^{2}\right)\right)+\frac{1}{36} b_{2} b_{3} c_{1}+\frac{1}{72} b_{3}^{2} \\
& -\frac{7}{144} b_{2} c_{1}\left(c_{1}^{2}+x\left(4-c_{1}^{2}\right)\right)-\frac{1}{36}\left(c_{1}^{2}+x\left(4-c_{1}^{2}\right)\right)^{2} \\
& +\frac{1}{32}\left(b_{2}+c_{1}\right)\left[c_{1}^{3}+2 c_{1}\left(4-c_{1}^{2}\right) x-c_{1}\left(4-c_{1}^{2}\right) x^{2}\right. \\
& \left.+2\left(1-|x|^{2}\right)\left(4-c_{1}^{2}\right) z\right] \mid \\
= & \mid \frac{1}{8}\left(b_{4}-b_{2} b_{3}\right) c_{1}+\frac{1}{8}\left(b_{3}-\frac{8}{9} b_{2}^{2}\right) c_{1}^{2}+\frac{1}{8}\left(b_{2} b_{4}-b_{3}^{2}\right) \\
& -\frac{1}{9}\left(b_{3}-\frac{9}{16} b_{2}^{2}\right) c_{1}^{2}-\frac{1}{9}\left(b_{3}-\frac{9}{16} b_{2}^{2}\right)\left(4-c_{1}^{2}\right) x+\frac{1}{36} b_{2} b_{3} c_{1} \\
& +\frac{1}{72} b_{3}^{2}-\frac{5}{288} b_{2} c_{1}^{3}+\frac{1}{288} c_{1}^{4}+\frac{1}{72} b_{2} c_{1}\left(4-c_{1}^{2}\right) x+\frac{1}{144} c_{1}^{2} x\left(4-c_{1}^{2}\right) \\
& -\frac{1}{36} x^{2}\left(4-c_{1}^{2}\right)^{2}-\frac{1}{32} c_{1} b_{2} x^{2}\left(4-c_{1}^{2}\right)-\frac{1}{32} c_{1}^{2}\left(4-c_{1}^{2}\right) x^{2} \\
& +\frac{1}{16}\left(b_{2}+c_{1}\right)\left(4-c_{1}^{2}\right)\left(1-|x|^{2}\right) z \mid
\end{aligned}
$$


By Lemma 1, we have $\left|c_{1}\right| \leq 2$. For convenience of notation, we take $c_{1}=c$ and we may assume without loss of generality that $c \in[0,2]$. Applying the triangle inequality in above equation with $\mu=|x|$ and using Lemma 3, Lemma 5, Lemma 7 and Lemma 8, we obtain

$$
\begin{aligned}
\left|a_{2} a_{4}-a_{3}^{2}\right| \leq & \frac{1}{8}\left|b_{4}-b_{2} b_{3}\right| c+\frac{1}{8}\left|b_{3}-\frac{8}{9} b_{2}^{2}\right| c^{2}+\frac{1}{8}\left|b_{2} b_{4}-b_{3}^{2}\right|+\frac{1}{9} \mid b_{3} \\
& -\frac{9}{16} b_{2}^{2}\left|c^{2}+\frac{1}{9}\right| b_{3}-\left.\frac{9}{16} b_{2}^{2}\left|\left(4-c^{2}\right) \mu+\frac{1}{36}\right| b_{2}|| b_{3}\left|c+\frac{1}{72}\right| b_{3}\right|^{2} \\
& +\frac{5}{288}\left|b_{2}\right| c^{3}+\frac{1}{288} c^{4}+\frac{1}{72}\left|b_{2}\right| c\left(4-c^{2}\right) \mu+\frac{1}{144} c^{2}\left(4-c^{2}\right) \mu \\
& +\frac{1}{36}\left(4-c^{2}\right)^{2} \mu^{2}+\frac{1}{32}\left|b_{2}\right| c\left(4-c^{2}\right) \mu^{2}+\frac{1}{32} c^{2} \mu^{2}\left(4-c^{2}\right) \\
& +\frac{1}{16}\left(\left|b_{2}\right|+c\right)\left(4-c^{2}\right)\left(1-\mu^{2}\right) \\
\leq & \frac{1}{4} c+\frac{1}{8} c^{2}+\frac{1}{8}+\frac{1}{9} c^{2}+\frac{1}{9}\left(4-c^{2}\right) \mu+\frac{1}{6} c+\frac{1}{8}+\frac{5}{144} c^{3} \\
& +\frac{1}{288} c^{4}+\frac{1}{36} c\left(4-c^{2}\right) \mu+\frac{1}{144} c^{2}\left(4-c^{2}\right) \mu+\frac{1}{36}\left(4-c^{2}\right)^{2} \mu^{2} \\
+ & \frac{1}{16} c\left(4-c^{2}\right) \mu^{2}+\frac{1}{32} c^{2} \mu^{2}\left(4-c^{2}\right)+\frac{1}{16}(2+c)\left(4-c^{2}\right)\left(1-\mu^{2}\right) \\
= & \frac{3}{4}+\frac{2}{3} c+\frac{1}{9} c^{2}-\frac{1}{36} c^{3}+\frac{1}{288} c^{4}+\mu\left(4-c^{2}\right)\left(\frac{1}{9}+\frac{1}{36} c+\frac{1}{144} c^{2}\right) \\
& +\frac{1}{288}\left(c^{2}-4\right)\left(4-c^{2}\right) \mu^{2}=F_{2}(c, \mu)
\end{aligned}
$$

Differentiating $F_{2}(c, \mu)$ in above equation with respect to $\mu$, we get

$$
\begin{aligned}
\frac{\partial F_{2}}{\partial \mu} & =\left(\frac{1}{9}+\frac{1}{36} c+\frac{1}{144} c^{2}\right)\left(4-c^{2}\right)+\frac{1}{144}\left(c^{2}-4\right)\left(4-c^{2}\right) \mu \\
& =\left(\frac{1}{36}(4-\mu)+\frac{1}{36} c+\frac{1}{144} c^{2}+\frac{1}{144} \mu c^{2}\right)\left(4-c^{2}\right)>0 \text { for } \quad 0 \leq \mu \leq 1 .
\end{aligned}
$$

Therefore $F_{2}(c, \mu)$ is an increasing function of $\mu$ for $0 \leq \mu \leq 1$ and for any fixed $c$ with $c \in[0,2]$. Hence it attains maximum value at $\mu=1$. Thus

$$
\max _{0 \leq \mu \leq 1} F_{2}(c, \mu)=F_{2}(c, 1)=G_{2}(c) \text { (say). }
$$

Therefore from (15) and (16), we have

$$
\mathrm{G}_{2}(\mathrm{c})=\frac{1}{144}\left(164+112 \mathrm{c}+8 \mathrm{c}^{2}-8 \mathrm{c}^{3}-\mathrm{c}^{4}\right) .
$$


Now

$$
\begin{aligned}
\mathrm{G}_{2}^{\prime}(\mathrm{c}) & =\frac{1}{36}\left[28+4 \mathrm{c}-6 \mathrm{c}^{2}-\mathrm{c}^{3}\right] \\
& =\frac{1}{36}\left[4+(6+\mathrm{c})\left(4-\mathrm{c}^{2}\right)\right]>0 \quad \text { for } \quad \mathrm{c} \in[0,2] .
\end{aligned}
$$

This shows that $G_{2}(c)$ is an increasing function of $c$, hence it will attains maximum value at $c=2$. Therefore

$$
\max _{0 \leq \mathrm{c} \leq 2} \mathrm{G}_{2}(\mathrm{c})=\mathrm{G}_{2}(2)=\frac{85}{36} \text {. }
$$

Hence the upper bound on $\left|a_{2} a_{4}-a_{3}^{2}\right|$ can bee obtained by setting $\mu=1$ and $c=2$ in (15) or $c=2$ in (17), therefore

$$
\left|a_{2} a_{4}-a_{3}^{2}\right| \leq \frac{85}{36}
$$

Theorem 3 Let the function $\mathrm{f} \in \mathcal{C}$ be given by (1), then

$$
\left|\mathrm{H}_{3}(1)\right| \leq \frac{289}{12} \text {. }
$$

Proof. Let $f \in \mathcal{C}$ of the form (1), then by definition $\mathrm{H}_{3}(1)$ is given by

$$
\begin{aligned}
H_{3}(1) & =\left|\begin{array}{lll}
a_{1} & a_{2} & a_{3} \\
a_{2} & a_{3} & a_{4} \\
a_{3} & a_{4} & a_{5}
\end{array}\right| \\
& =a_{3}\left(a_{2} a_{4}-a_{3}^{2}\right)-a_{4}\left(a_{4}-a_{2} a_{3}\right)+a_{5}\left(a_{3}-a_{2}^{2}\right) .
\end{aligned}
$$

Using the triangle inequality in (19), we have

$$
\left|H_{3}(1)\right|=\left|a_{3}\right|\left|a_{2} a_{4}-a_{3}^{2}\right|+\left|a_{4}\right|\left|a_{4}-a_{2} a_{3}\right|+\left|a_{5}\right|\left|a_{3}-a_{2}^{2}\right| .
$$

Now applying Lemma 4, Lemma 6, Theorem 1 and Theorem 2 in (20), we finally have the bound on $\mathrm{H}_{3}(1)$ as desired.

\section{Acknowledgment}

The authors are grateful to the referees for the valuable comments. Also, the present investigation of second author is supported by Department of Science and Technology, New Delhi, Government of India under Sanction Letter No. SR/FTP/MS-015/2010. 


\section{References}

[1] K. O. Babalola, On $\mathrm{H}_{3}$ (1) Hankel determinant for some classes of univalent functions, Inequal. Theory Appl., 6 (2007), 1-7.

[2] D. Bansal, Upper bound of second Hankel determinant for a new class of analytic functions, Appl. Math. Lett., 26 (1) (2013), 103-107.

[3] D. Bansal, S. Maharana, J. K. Prajapat, Third order Hankel Determinant for certain univalent functions, J. Korean Math. Soc., 52 (6) (2015), $1139-1148$.

[4] C. Carathéodory, Über den variabilitätsbereich der Fourier'schen Konstanten Von Positiven harmonischen Funktionen, Rend. Circ. Mat. Palermo, 32 (1911), 193-217.

[5] P. L. Duren, Univalent Functions, vol. 259 of Grundlehren der Mathematischen Wissenschaften, Springer-Verlag, New York, Berlin, Heidelberg, Tokyo, 1983.

[6] R. Ehrenborg, The Hankel determinant of exponential polynomials, Amer. Math. Monthly, 107 (2000), 557-560.

[7] M. Fekete, G. Szegö, Eine Benberkung uber ungerada Schlichte funktionen, J. London Math. Soc., 8 (1933), 85-89.

[8] W. K. Hayman, On second Hankel determinant of mean univalent functions, Proc. Lond. Math. Soc., 18 (1968), 77-94.

[9] W. Kaplan, Close-to-convex schlicht functions, Mich. Math. J., 1 (1952), 169-185.

[10] F. R. Keogh, E. P. Merkes, A coefficient inequality for certain classes of analytic functions, Proc. Amer. Math. Soc., 20 (1969), 8-12.

[11] W. Koepf, On the Fekete-Szegö problem for close-to-convex functions, Proc. Amer. Math. Soc., 101 (1987), 89-95.

[12] A. Janteng, S. Halim, M. Darus, Coefficient inequality for a function whose derivative has a positive real part, J. Inequal. Pure Appl. Math., 7 (2) (2006), Article 50. 
[13] S. K. Lee, V. Ravichandran, S. Supramaniam, Bounds for the second Hankel determinant of certain univalent functions, J. Inequal. Appl., 2013 (2013), Article 281.

[14] R. J. Libera, E. J. Zlotkiewicz, coefficient bounds for the inverse of a function with derivative in $\mathcal{P}$, Proc. Amer. Math. Soc., 87 (2) (1983), $251-257$.

[15] R. J. Libera, E. J. Zlotkiewicz, Early coefficients of the inverse of a regular convex function, Proc. Amer. Math. Soc., 85 (2) (1982), 225230.

[16] J. W. Noonan, D. K. Thomas, On the second Hankel determinant of areally mean p-valent functions, Trans. Amer. Math. Soc., 223 (2) (1976), $337-346$.

[17] K. I. Noor, Hankel determinant problem for the class of function with bounded boundary rotation, Rev. Roum. Math. Pures Et Appl. 28 (1983), 731-739.

[18] K. I. Noor, S. A. Al-Bany, On Bazilevic functions, Int. J. Math. Math. Sci., 10 (1) (1987), 79-88.

[19] K. I. Noor, On analytic functions related with function of bounded boundary rotation, Comment. Math. Univ. St. Pauli, 30 (2) (1981), 113118.

[20] K. I. Noor, Higer order close-to-convex functions, Math. Japon, 37 (1) (1992), 1-8.

[21] C. Pommerenke, On the Hankel determinants of univalent functions, Mathematika, 14 (1967), 108-112.

[22] C. Pommerenke, On the coefficients and Hankel determinant of univalent functions, J. Lond. Math. Soc., 41 (1966), 111-,112.

[23] M. O. Reade, On close-to-convex functions, Mich. Math. J., 3 (1955-56), $59-62$.

[24] M. Raza, S. N. Malik, Upper bound of the third Hankel determinant for a class of analytic functions related with Lemniscate of Bernoulli, $J$. Inequal. Appl., 2013 (2013), Article 412. 